\title{
Microscopic Theory of Transconductivity
}

\author{
A. P. JAUHO, M. B. BØNSAGER ${ }^{*}$, K. FLENSBERG ${ }^{\dagger}$, B. Y.-K. HU and J. KINARET ${ }^{*}$ \\ MIC, Technical University of Denmark, Bldg 345east. DK-2800 Lyngby, Denmark \\ Nordita, Blegdamsvej 17, DK-2100 Copenhagen $\emptyset$, Denmark
}

\begin{abstract}
Measurements of momentum transfer between two closely spaced mesoscopic electronic systems, which couple via Coulomb interaction but where tunneling is inhibited, have proven to be a fruitful method of extracting information about interactions in mesoscopic systems. We report a fully microscopic theory for transconductivity $\sigma_{12}$, or, equivalently, momentum transfer rate between the system constituents. Our main formal result expresses the transconductivity in terms of two fluctuation diagrams, which are topologically related, but not equivalent to, the Azlamazov-Larkin and Maki-Thompson diagrams known for superconductivity. In the present paper the magnetic field dependence of $\sigma_{12}$ is discussed, and we find that $\sigma_{12}(B)$ is strongly enhanced over its zero field value, and it displays strong features, which can be understood in terms of a competition between density-of-states and screening effects.
\end{abstract}

Consider two systems containing mobile charge carriers so close to each other that the charges in the two respective subsystems feel the Coulomb forces originating from the other subsystem, and yet far enough away from each other that direct charge transfer between the two subsystems is not possible. Experimental realizations of such systems are. for example. Coulomb coupled double quantum well systems, [1,2] arrangements where a $3 \mathrm{D}$ system is close to a $2 \mathrm{D}$ system, [3] or two nearby quantum wires. A scattering event between a carrier in one system and a carrier in the other system leads to momentum transfer between the two subsystems. Thus, if a current is driven through one of the systems (henceforth the driven system is denoted as layer 1), then an induced current is dragged in the other subsystem (layer 2). Alternatively, if no current is allowed to flow in layer 2, a voltage is induced. Due to momentum conservation the two particle number currents flow in the same direction. Since the mechanism for the Coulomb drag is carrier-carrier scattering the drag current is proportional to the square of the effective interaction between the subsystems. The available phase space for electron-electron scattering tends to zero at low temperatures, and consequently one expects Coulomb drag to decrease with decreasing temperature. At low temperatures, the two Pauli factors entering the carrier-carrier scattering rate lead to a $T^{2}$-dependence, and this behavior is approximately seen in experiments. [1] Note, however, that there are small, but important deviations from the simple $T^{2}$-law; these deviations have been the topic of much recent interest. $[1,4,5]$

The simplest possible theoretical description for Coulomb drag is based on the Boltzmann equation.

\footnotetext{
* Present address: Department of Physics, Indiana University, Bloomington, Indiana 47405-4202

$\uparrow$ Present address: Danish Institute of Fundamental Metrology, DK-2800 Lyngby, Denmark

$\doteqdot$ Present address: Chalmers University of Technology, Göteborg, S-41296 Sweden
} 
Within this formulation the following expression can be derived for the drag rate [6]:

$$
\begin{aligned}
\frac{1}{\tau_{D}}=\frac{\hbar^{2}}{2 \pi^{2} n_{1} m k T} & \int_{o}^{\infty} d q \int_{o}^{\infty} d \omega q^{3}|e \phi(q, \omega)|^{2} \\
& {\left[\operatorname{Im} \chi_{0}(q, \omega)\right]^{2} \frac{1}{\sinh ^{2}(\hbar \omega / 2 k T)} . }
\end{aligned}
$$

Here $\phi(q, \omega)=\phi(q) / \epsilon(q, \omega)$ is the dynamically screened interaction between carriers in the two layers, and $\chi \mathrm{O}(q, \omega)$ is the bare RPA polarization function. The above expression is valid only in the weakscattering limit, and a generalization is needed if one wishes to examine, say, weak-localization corrections or effects of external magnetic field. Only a fully microscopic theory can address these questions, and we have recently carried out such an analysis [7.8]. The calculations are based on the Kubo formula, which expresses the transconductance (and hence the drag rate) in terms of a retarded current-current correlation function,

$$
\begin{aligned}
& \sigma_{i j}^{\alpha \beta}(x-x ; \Omega)=\frac{i e^{2}}{\Omega} \Pi_{i j}^{\alpha \beta, r}\left(x-x^{\prime} ; \Omega\right) \\
& \quad+\frac{i e^{2}}{m \Omega} \delta\left(x-x^{\prime}\right) \delta_{i j} \delta_{\alpha \beta} \rho_{i}(x) .
\end{aligned}
$$

where (throughout we use $\hbar=1$ )

$$
\Pi_{i j}^{\alpha \beta, r}\left(x-x^{\prime} ; t-t^{\prime}\right)=-i \Theta\left(t-t^{\prime}\right)\left\langle\left[j_{i}^{\alpha}(x, t), j_{j}^{\beta}\left(x^{\prime}, t^{\prime}\right)\right]\right\rangle .
$$

Here $\{i j\}$ indicate the subsystem, $\{\alpha \beta\}$ in the superscripts label the Cartesian coordinates, $\Omega$ is the external frequency, $\rho_{i}(x)$ is the particle density in subsystem $i$, and $j(x, t)$ is the particle current operator. The analysis proceeds via a systematic expansion in the interlayer interaction, and one finds in second order $[7,8]$

$$
\begin{gathered}
\sigma_{21}^{\alpha \beta}=\frac{-e^{2}}{2 \hbar^{3} V} \sum_{q} \int_{-\infty}^{\infty} \frac{d \omega}{2 \pi}|\phi(q, \omega)|^{2} \frac{\partial n_{B}(\hbar \omega)}{\partial \omega} \\
\times \triangle_{2}^{\alpha}(q, q ; \omega+i \delta, \omega-i \delta) \triangle_{1}^{\beta}(-q,-q ;-\omega-i \delta,-\omega+i \delta),
\end{gathered}
$$

where $\phi(q, \omega)$ is the dynamically screened interaction and $\Delta$ is a three-body correlation function [7],

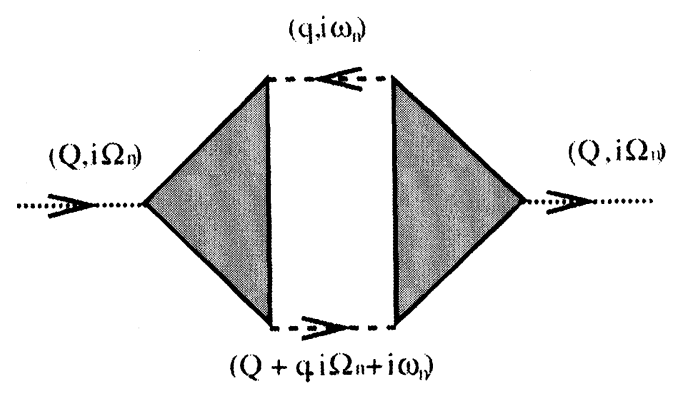

FIGURE 1 Diagram determining transconductivity in terms of triangle functions

$$
\begin{aligned}
\Delta\left(q, q ; i \omega_{n}, i \omega_{n}^{\prime}\right)=\int_{0}^{\beta} d \tau \int_{0}^{\beta} d \tau^{\prime} e^{i \omega_{n} \tau} e^{i \omega_{n}^{\prime} \tau^{\prime}} i \\
\left\langle T_{\tau} j(q=0, \tau=0) \rho(q, \tau) \rho\left(-q, \tau^{\prime}\right)\right\rangle .
\end{aligned}
$$

The analytic continuation from the Matsubara frequencies $i \omega_{n}$ to real frequencies is discussed in detail in Ref. [7]. Figure 1 illustrates the diagrammatic structure of the transconductivity. The shaded triangles (which correspond to three-body correlation functions) can be evaluated at different levels of approximation, and we have shown [7] that previously known results, obtained either by Boltzmann equations approach or memory functions [9] can be recovered. Very recently, a similar analysis has been presented by Kamenev and Oreg. [10]

In the lowest order approximation the bare triangle reduces to a product of three Green functions, and it thus appears to be in disagreement with the Boltzman result (1) which only involves RPA-bubbles, which consist of two Green functions. It is, however, possible to recover the connection with the following caveats: 1) For $B=0$ one must have momentumindependent transport relaxation times [7], and 2) $B \neq 0$ one must work in the high-field weak-scattering limit. The upshot of the analysis is that one can, if $\omega_{c} \tau \gg 1$, still use an expression which formally coincides with the Boltzmannian result (1). but with the following important modifications: 1) The polarization functions $\chi 0$ must be replaced with full $\chi$ 's, including the magnetic field and impurity effects. Thus one must solve the vertex equation for $\chi$ in a suitable conserving approximation (e.g. including ladder diagrams); and 2) The screened interaction must be 
calculated at the same level of approximation, i.e. including magnetic field and impurity effects.

A full description of our calculations is given elsewhere [8], and here we will give a qualitative description of the main physical results. First, the drag rate is significantly enhanced: our preliminary numerical results indicate that the enhancement factor is $60-70$. [11] Experimentally this is very important, since generally drag effects are small, and any enhancement is most welcome. Secondly, the drag rate displays strong structure whenever the Fermi level crosses a Landau level. Finally, the temperature dependence is quite dramatic; it is qualitatively different at different values of the magnetic field.

A physical understanding of these findings can be obtained with the help of Fig. 2. First, the large degeneracy of Landau levels enhances the density-ofstates at Fermi level enormously, and since the drag rate is proportional to $\operatorname{Im}^{2} \chi / \sinh ^{2}(\hbar \omega / 2 k T) \equiv$ $S(q, \omega) S(-q, \omega)$, where $S$ is the structure factor reflecting the available phase-space, an enhancement of the density-of-states necessarily leads to an increased drag rate.

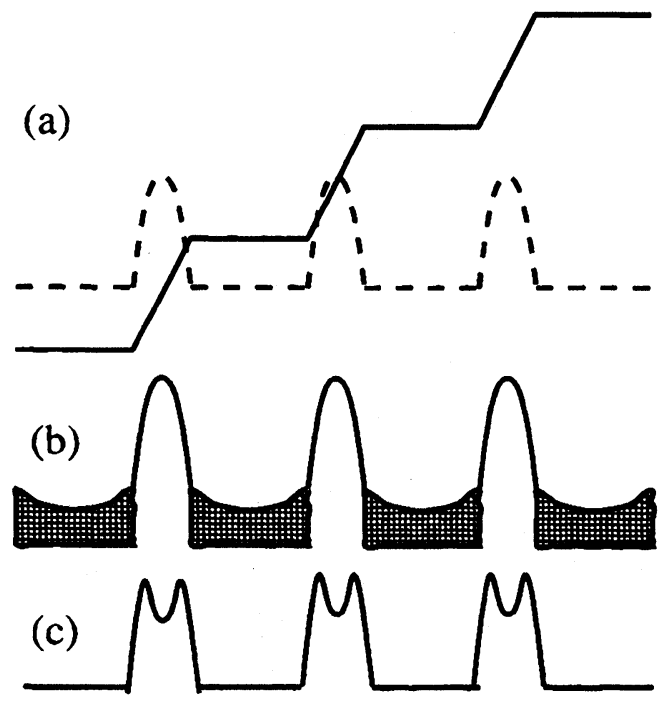

FIGURE 2. Transport coefficients in the Integer Quantum Hall regime (schematic). Legend: (a) Solid line: Hall conductivity in units of $e^{2} / h$, Dashed line: Longitudinal resistance (arb. units). (b) Density of states with localized states in hatched regions. (c) Predicted drag resistance
It is not surprising that the drag rate should show peaks as the the Fermi level moves through Landau levels as a function of magnetic field [12]. The situation is quite analogous with the "normal" longitudinal resistance observed in the integer quantum Hall regime (sketched in panel (a) of Figure 2): dissipative transport takes place only when the Fermi level lies within the extended states (unshaded areas in Fig. 2(b)). Thus, there is drag only when states at the Fermi level can undergo scatterings where momentum is exchanged between the layers. The structure within the predicted peaks in the drag rate has a more subtle explanation. In addition to the available phasespace the drag is also strongly influenced by the screened interaction. This, in turn, depends on the number of states participating in the screening process. Thus, when the Fermi level approaches the center of a Landau level. when the number of states approaches a maximum, the screening becomes more effective. and the interlayer coupling becomes weaker. Based on this picture one expects that the drag peaks may show internal structure, and this behavior is skteched in Fig. 2(c). Our numerical results [8] fully corroborate the above discussion. The possibility of direct observation of carrier-carrier interaction related effects in a transport quantity such as the drag rate is quite unusual, and should be of significant interest to experimentalists.

\section{References}

[1] T. J. Gramila, J. P. Eisenstein, A. H MacDonald, L. N. Pfeiffer, and K. W. West, Phys. Rev. Lett. 66, 1216 (1991) ; Phys. Rev. B 47, 12957 (1993); Physica B 197, 442 (1994).

[2] U. Sivan, P. M. Solomon, and H. Shtrikman, Phys. Rev. Lett. 68, 1196 (1992).

[3] P. M. Solomon, P. J. Price, D. J. Frank, and D. C. L. Tulipe, Phys. Rev. Lett. 63, 2508 (1989).

[4] H. C. Tso and P. Vasilopoulos and F. M. Peeters, Phys. Rev. Lett. 68, 2516 (1992); ibid. 70, 2146 (1993); H. C. Tso and P. Vasilopoulos, Phys. Rev. B 45, 1333 (1992).

[5] K. Flensberg and B. Y.-K. Hu, Phys. Rev. Lett. 73, 3572 (1994).

[6] A.-P. Jauho and H. Smith, Phys. Rev. B 47, 4420 (1993).

[7] K. Flensberg, B. Y.-K. Hu, A.-P. Jauho, and J. M. Kinaret, Phys. Rev. B 52, 14761 (1995)

[8] M. C. Bønsager, B. Y. -K. Hu, K. Flensberg, and A.-P. Jauho, (unpublished)

[9] L. Zheng and A. H. MacDonald, Phys. Rev. B 48, 8203 (1993).

[10] A. Kamenev and Y. Oreg, Phys. Rev. B 527516 (1995) 
[11] The enhancement factor is consistent with recent experiments at Cambridge, U.K. We are grateful to Dr. N. Hill for communicating us his unpublished data.

[12] Here we restrict ourselves to the case when the densities of the two gases are same: under these conditions the drag effects are most pronounced.

\section{Biographies}

A. P. Jauho M. Sc. 1975 and Lic. Techn. 1979 (Helsinki University of Technology), Ph. D. 1982 (Cornell University); post-doc Nordita 1981-1984, Chalmers University 1984-1985, faculty at Copenhagen University 1985-1992, faculty at Nordita 1992-1993, Leader of Theory Group at Mikroelektronik Centret, Technical University of Denmark 1992 - present
M. Bønsager M. Sc. 1995 (Technical University of Denmark), Graduate Student at Indiana University, Bloomington 1995 - present

B. Y.-K. Hu B.A. 1984 (Swarthmore College), M.Sc. 1987 (Cornell University), Ph.D. 1990 (Cornell University), post-doc U. of Maryland 1990-1993. research scientist MIC, DTU 1993 - present

K. Flensberg M. Sc. 1987 (DTU), Ph. D. 1989 (DTU). post-doc. U. of Tennessee 1989-91. Nordita 1991-93, Carlsberg Research Fellow 1993-95. Staff Scientist, Danish Institute for Fundamental Metrology, 1995 - present 

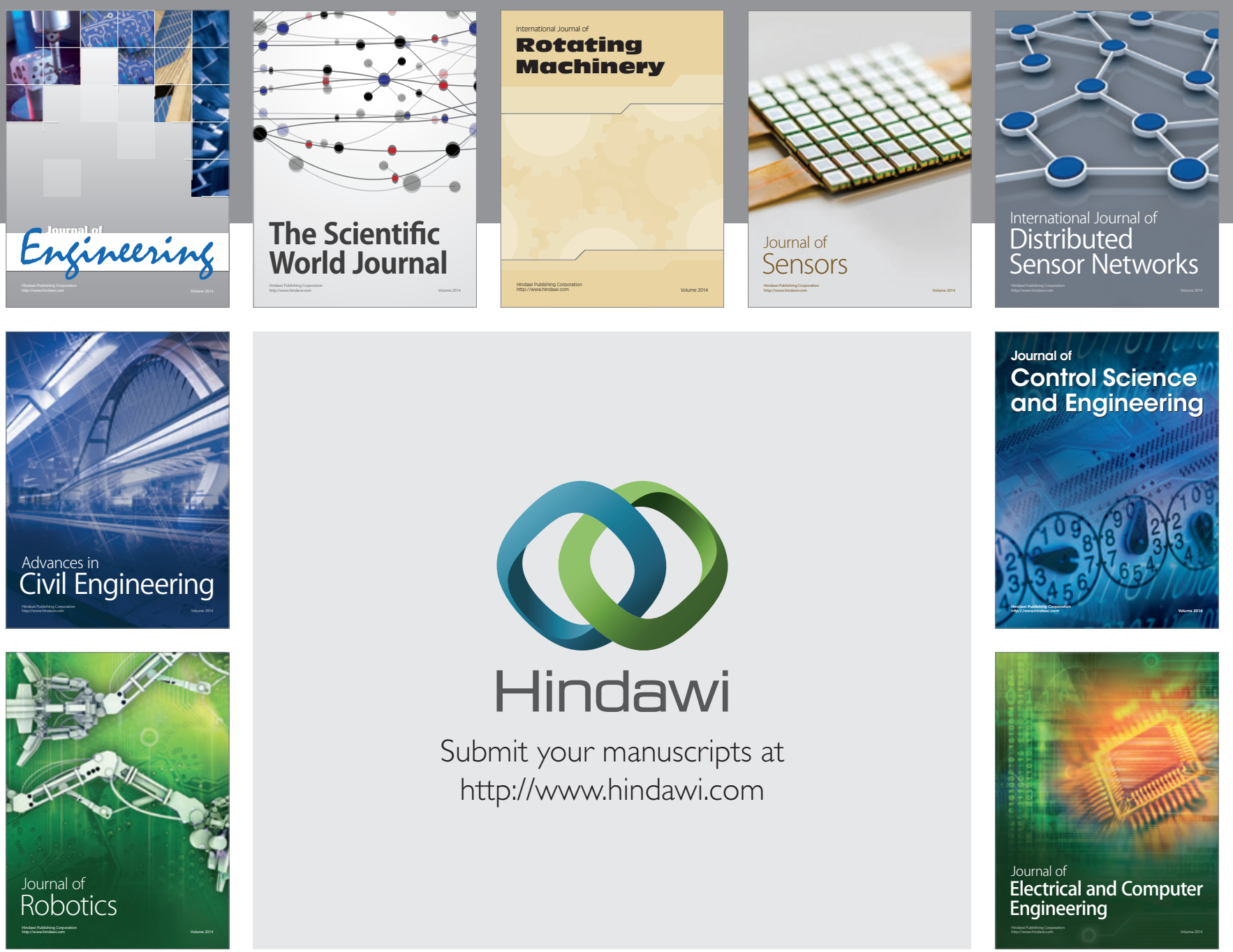

Submit your manuscripts at

http://www.hindawi.com
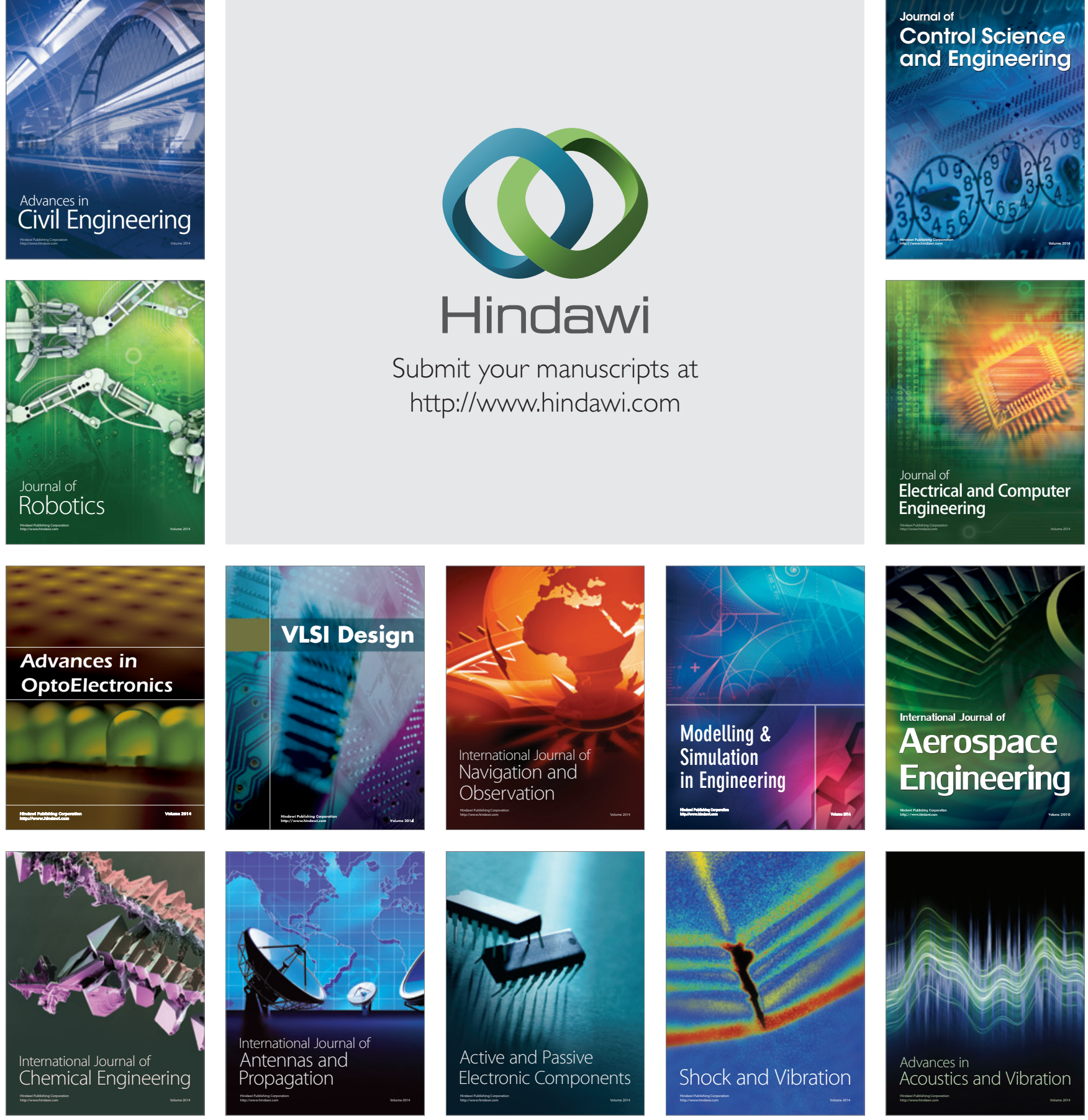